\title{
Economic, environmental and mixed objective functions in non-linear process optimization using simulated annealing and tabu search
}

\author{
Florinda Martins, Carlos A.V. Costa
}

ABSTRACT

\begin{abstract}
Screening of topologies developed by hierarchical heuristic procedures can be carried out by comparing their optimal performance. In this work we will be exploiting mono-objective process optimization usingtwo algorithms, simulated annealing and tabu search, and four different objective functions: two of the net present value type, one of them including environmental costs and two of the global potential impacttype. The hydrodealkylation of toluene to produce benzene was used as case study, considering five topologies with different complexities mainly obtained by including or not liquid recycling and heat integration.

The performance of the algorithms together with the objective functions was observed, analyzed and discussed from various perspectives: average deviation of results for each algorithm, capacity for produc- ing high purity product, screening of topologies, objective functions robustness in screening of topologies, trade-offs between economic and environmental type objective functions and variability of optimum solutions.
\end{abstract}

Keywords: Simulated annealing, Tabu search, Economic objective function, Environmental objective function

\section{Introduction}

When using some heuristic hierarchical procedures for conceptual process design (Douglas, 1988; Smith, 1995), the result is a set of feasible flowsheets that can be further quantified and developed by using a process simulator and optimized and screened by comparison of their optimal performance in terms of some criterion (objective function). With this procedure (comparison included) it is possible to determine the global optimal solution that will allow the screening of the several feasible flowsheets as the performance of each flowsheet can be compared with the optimum.

Full flowsheet optimization problems are constituted by a set of linear and/or non-linear equations, related with equipment design and/or mass balance equations solution, with multiple integer and/orcontinuous variables. These types of problems arecombinatorial in nature due to the huge number of possiblecombinations of the multiple variables values that can solve that set of equations. Due to this combinatorial nature meta-heuristics are algorithms that can be suitable to solve these problems although not being able to guarantee global optimality but many times conducting to very good solutions. Among these algorithms, simulated anneal- ing (SA) and tabu search (TS), can be good candidates. They were chosen because they are potentially suitable for solving this kind of problems, they are quite different which will allow the comparison of the application of these different meta-heuristics to this type of problems and because there is almost no experience in applying these algorithms to full flowsheet optimizations problems. Population search algorithms are another possibility that was not considered in this work particularly because they can put premature convergence problems which deserve the development of similar indicators that were out of the scope of this research (Hertz \& Widmer, 2003).

Historically, SA was successfully applied to solve the salesman problem and in the design of integrated complex circuits and its implementation is relatively simple (Press, Teukolsky, Vetterling, \& Flannery, 1992). In the chemical engineering field some applications can be mentioned like in heat exchange networks (Dolan, Cummings, \& Van, 1990), in batch process scheduling (Cardoso, Salcedo, Azevedo, \& Barbosa, 1997; Das, Cummings, \&LeVan, 1990; Ku and Karimi, 1991; Patel, Mah, \& Karimi, 1991), in the optimization of batch distillation processes (Hanke and Li, 2000) and in dynamic optimization (Faber et al., 2005). In what concerns optimization problems only involving continuous variables one can mention the works of Press and Teukolsky (1991) and Cardoso, Salcedo, and Azevedo (1996).

TS was applied by Cavin, Fischer, Mosat, and Hungerbuhler (2005) in batch process scheduling and by Lin and Miller (2004) 


\section{Nomenclature}

C total annual cost (D)

$C_{A} \quad$ global potential environmental impact cost (D)

$C_{M} \quad$ worst value of the objective function

C difference between values of the objective function in two consecutivesiterations

d depreciation

GPEI global potential environmental impact

GPEI-EPS global potential environmental impact (EPS methodology)

$i \quad$ interest rate

I total capital investment (D)

$k \quad$ actual iteration number in TS algorithm

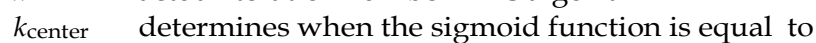
0.5

M total number of iterations in TS algorithm

$n \quad$ service life (year)

NPV1 net present value (D)

NPV2 net present value with environmental costs (D)

$P \quad$ probability

$T_{S A} \quad$ temperature on SA algorithm

$T_{S A 0} \quad$ initial temperature on SA algorithm

$V \quad$ annual income (D )

vc optimization variable

vc vector optimization variables random increments

$\mathrm{vc}(0) \quad$ fresh reactant molar ratio

$\mathrm{vc}(3) \quad$ flash pressure $(\mathrm{Pa})$

$\mathrm{vc}(4)$ benzene molar fraction in the purge

$\mathrm{vc}(5) \quad$ purge flowrate $(\mathrm{mol} / \mathrm{s})$

$\mathrm{vc}(6) \quad$ reactor volume $\left(\mathrm{m}^{3}\right)$

$\mathrm{vc}(7) \quad$ column 2 top stream toluene molar fraction or benzene recovery

vc(8) column 2 bottom stream benzene molar fraction or toluene recovery

$\mathrm{vc}(9) \quad$ column 2 operating pressure $(\mathrm{Pa})$

$\mathrm{vc}(10)$ column 1 top stream benzene molar fraction or methane recovery

$\mathrm{vc}(11)$ column 1 reflux ratio

vc(12) column 1 operating pressure $(\mathrm{Pa})$

$\mathrm{vc}(13) \quad$ column 3 top stream toluene recovery

vc(14) column 3 operating pressure $(\mathrm{Pa})$

$\mathrm{vc}(15)$ column 1 number of trays

$\mathrm{vc}(16)$ column 2 number of trays

$\mathrm{vc}(18) \quad$ column 3 number of trays

vc(19) column 3 bottom stream diphenyl recovery

\section{Greek letters}

$\begin{array}{ll}- & \text { SAalgorithmparameter } \\ \text { o } & \text { TSalgorithm parameter } \\ \circ & \text { income tax }\end{array}$

Acronyms

A topology with one column without recirculation of the toluene/diphenyl stream and without energy integration

Ae similar to topology A but with energy integration

E topology with two columns without recirculation of the toluene/diphenyl stream and without energy integration

Ee similar to topology E but with energy integration

EPS Environmental Priority Strategies

Ge topology with three columns, recirculation of the toluene/diphenyl stream and energy integration
GPI global potential impact

LP linear problem

NLP non-linear problem

SA simulated annealing

TS tabu search

in the optimization of some case studies of chemical engineering, namely heat exchange networks and pump systems.

Theexperiencein applyingSA tofull process optimization problems even of the LP or NLP type is relatively scarce and there is practically no experience with TS.

One of the objectives of this paper is then to analyze and compare the performance of these two algorithms in the optimization of flowsheets of different complexities essentially resulting from more or less mass and/ or heat integration (reusing or recycling) for the same casestudy.

Pollution prevention is nowadays an issue of concern implying the consideration of environmental aspects since the conceptual and preliminary process design steps, as a way to develop plants withless environmental impact but withoutlosing economic competitiveness. Brennecke and Stadtherr (2002) identified some pollution prevention strategies and Cano-Ruiz and McRae (1998) presented a review on the strategies to incorporate environmental aspects in the design of processes. Some researchers although using economic type objective functions dealt with mass and/or energy conservation issues (El-Halwagi and Manousiouthakis, 1990). Dietz, Azzaro-Pantel, Pibouleau, and Domenech (2006) applied multiobjective optimization for multiproduct batch plant design using economic and environmental considerations.

In terms of process optimization the number of works that include environmental concerns in the objective function is also relatively scarce.

In this work we will be exploiting mono-objective process optimization using different objective functions looking for the traditional economic based optimum and toan optimum only based on environmental considerations, detecting eventual trade-offs existing between bothcriteria. Another approachisalsoexplored aiming at solving these trade-offs by using a mixed type objective function, e.g., economic plus environmental costs. So objective functions of the economic, environment and mixed type were used, namely net presentvalue(NPV), global potential environmental impact(GPEI) and mixed (NPV+ environmental costs).

So in this work we compare the performance of SA and TSfor different complexity flowsheets of the same case study and different objective functions and we compare and discuss the optimal conditions obtained.

\section{Algorithms and objective functions}

To perform process optimization we need a model, usually represented by a set of non-linear algebraic equations, a numerical algorithm to solve it, an optimization algorithm, boundaries for the search space and an objective function.

\subsection{Simulator}

Instead of writing down all the equations to represent the full process model we preferred the use of a commercial simulator, PROII (SimScience, 2005). This is easier than constructing and solving the "raw model" although presenting two main difficulties. The first one is the need for an interface between this type of simulator and the optimization algorithm. The second is linked to the identification of the causes for simulations failure. 
Since the optimization programme has no direct access to the modular simulator an interface was developed to transfer data between them. The optimization programmes were constructed in Visual Basic for Applications (VBA) and are composed by several modules:

- main

- introduction of data(optimizationalgorithmparameters, number of variables, choice of objective function, etc.)

- generation of next solutions

- construction of simulator input files

- simulator call and execution of simulation

- analysis 1: code errors in simulations
- extraction of output results

- analysis 2: impossibilities analysis (ex.: no existence of a stream flow, equipment construction impossibilities, like column diameter higher/smaller than what is usually constructed/used, etc.)

- economic calculations

- environmental calculations

- objective function calculation

- optimization algorithms calculations

Although no unit operation can disappear (that would change the topology) and since it is considered in the simulator input file (except pumps, because a check is done after the generation of the next solution; if it is not necessary it will be not be considered).

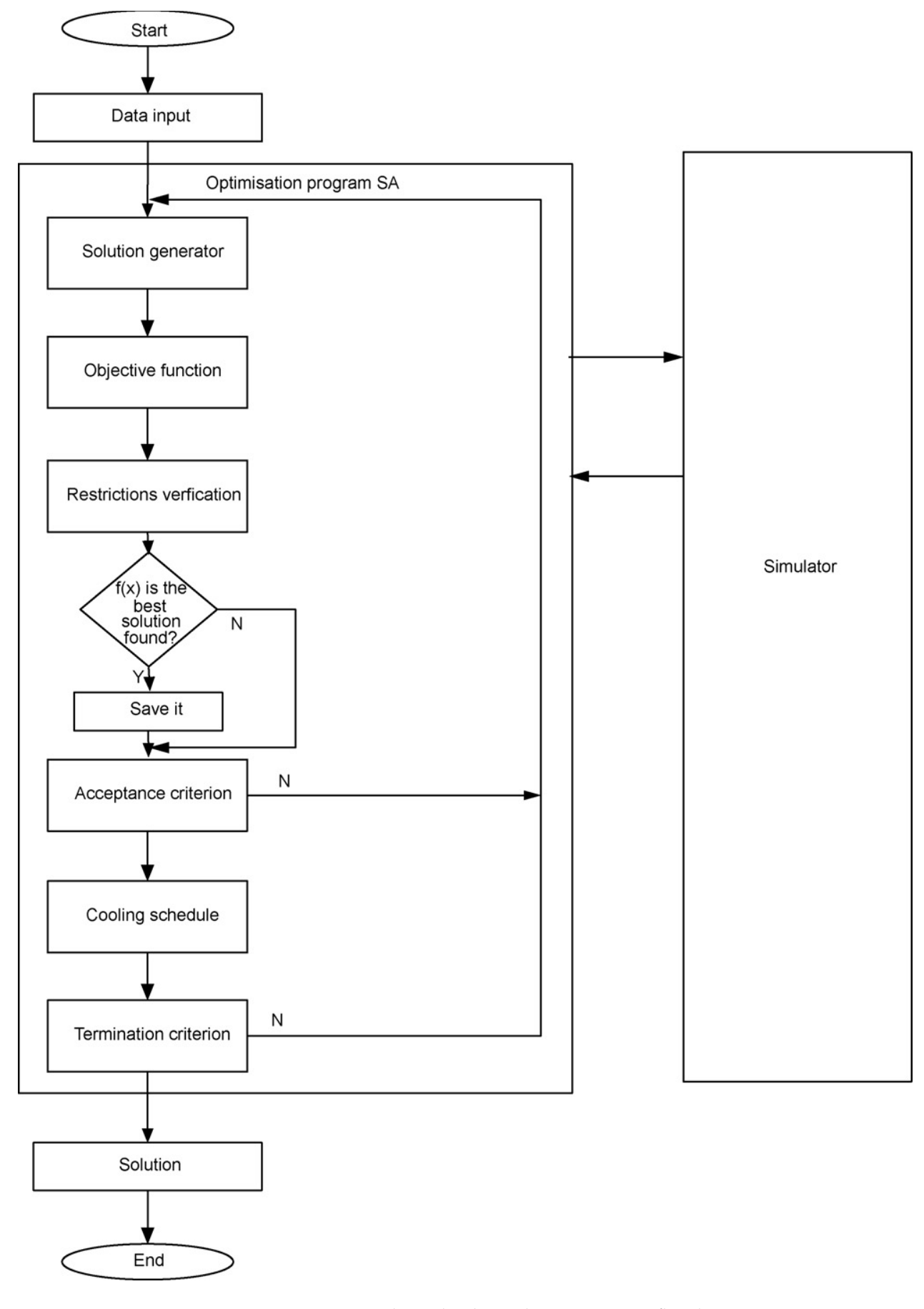

Fig. 1. Optimization with simulated annealing. Programme flowchart. 
Each iteration solution (if feasible) and almost every data/results related are automatically saved in an excel worksheet.

\subsection{Algorithms}

\subsubsection{Simulated annealing}

This multivariable algorithm was proposed by Kirkpatrick, Gelatt, and Vecchi (1983), being based on a random search, conditioned by a probabilistic function (e.g. Boltzman distribution), capable of exploring the whole solution space, avoiding local optima and reaching solutions independent from the starting one. Fig. 1 depicts the flowchart of the programme that involves several steps. Some more interesting details are described next.
In order to apply SA to a problem it is necessary to perform the following steps:

- define the objective function;

- determine the initial temperature;

- define the strategy to determine the next solution

- define the acceptance criteria and the annealing cooling schedule; - define the termination criteria.

When applying SA to a given problem a set of preliminary optimizations should be done in order to establish the value of some of the algorithm parameters, like initial temperature, the value of the constant in the annealing cooling schedule, the number of cycles in the termination criteria, etc. This is called the tuning

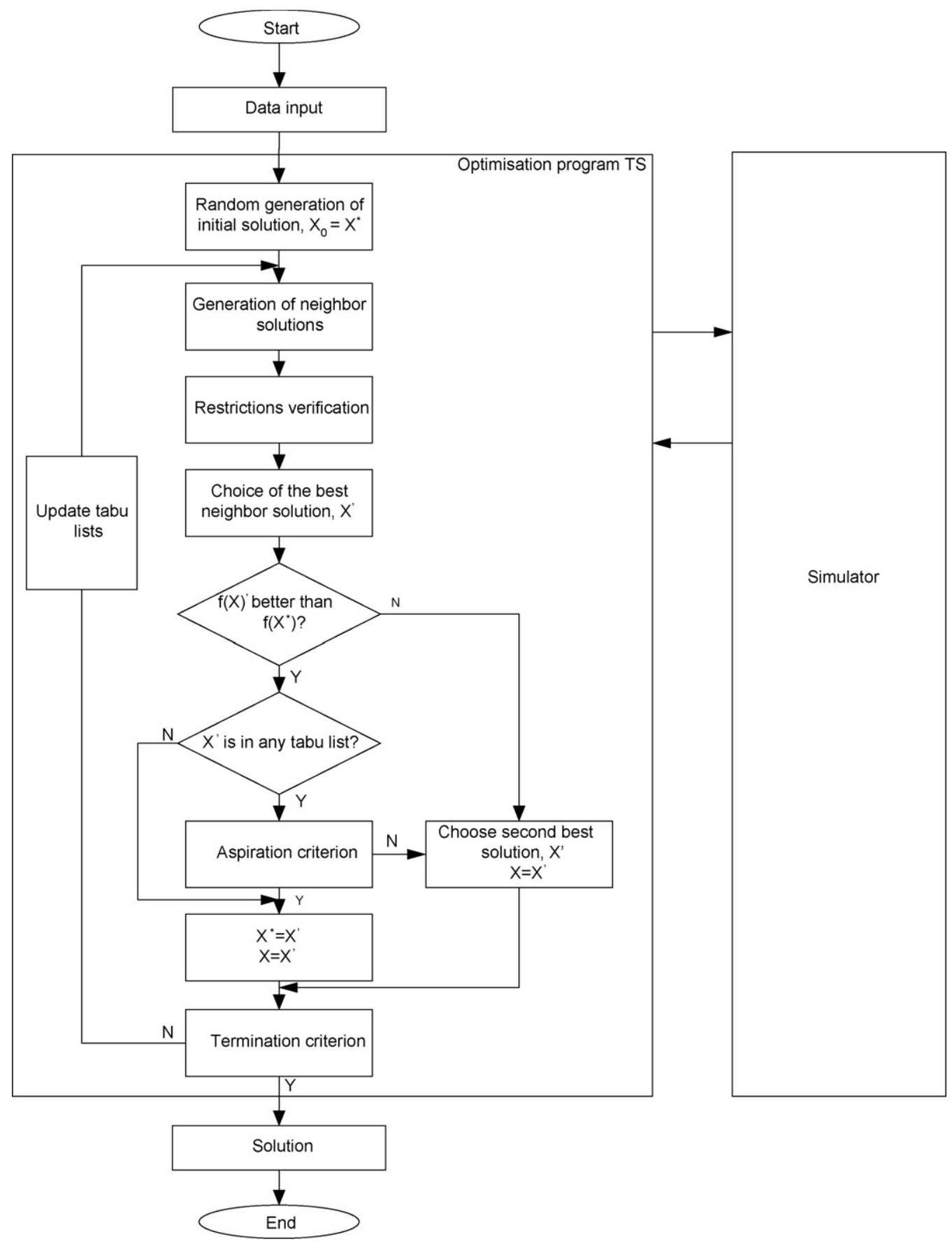

Fig. 2. Optimization with tabu search. Programme flowchart. 
phase. More details on SA algorithm can be found in Press et al. (1992).

\subsection{Initial temperature}

This temperature can be established by deciding which fraction of the generated solutions will be initially accepted. Larger temperatures imply the acceptance of more moves and a better exploitation of the search space but also larger computational time (Patel et al., 1991). We considered the initial temperature $\left(T_{S A 0}\right)$ as a function of the worst value of the objective function $\left(C_{M}\right)$ (Pibouleau, Domenech, Davin, \& e Azzaro-Pantel, 2005). Some preliminary optimizations were done considering three possibilities, namely $T_{S A 0}=0.01 \times C_{M}, T_{S A 0}=0.1 \times C_{M}$ and $T_{S A 0}=1 \times C_{M}$. After analysing the results, using the deviation between the actual solution to the best one, we concluded that $T_{S A 0}=0.1 \times C_{M}$ is a good choice.

\subsubsection{Strategy to determine the nextsolution}

Next solution can be obtained by perturbation of the value of all or part of the optimization variables depending on the problem. These perturbations may be random or heuristic (Press et al., 1992).

In the case of a chemical process with fixed topology there are continuous variables like the temperature and pressure and integer variables like the number of stages in the columns. For each iteration and optimization variable a random number is generated, that will correspond to a certain percentage of the variation range of that variable. The value of each optimization variable is then the sum of its minimum value and the randomly calculated increment. In the case of integer variables that value is rounded to the closest integer; when it exceeds the maximum value a new increment is randomly calculated. By doing this some restrictions are incorporated in the search space. The first solution was always viable and randomly generated.

\subsubsection{Acceptance criteria and annealing cooling schedule}

TheMetropolisalgorithmwaschosen becauseitusually displays better performance than the Glauber algorithm as can be seen in the works of Cardoso, Salcedo, and Azevedo (1994), Das et al. (1990) and Patel et al. (1991).

In the Metropolis algorithm the acceptance criteria for minimization is asfollows:

- accept the move if the value of the objective function decreases, since the new solution is better than the previous one;

- If the value of the objective function increases the new solution will be accepted with probability $P$, given by the Boltzman distribution (Press et al., 1992):

$$
P=\mathrm{e}^{\left(-\Delta C / T_{S A}\right)}
$$

where $P$ is the probability (between 0 and 1 ), $C$ is the difference between the values of the objective function in two consecutive iterations and $T_{S A}$ is the temperature. Nevertheless we always kept in memory the best solution ever found.

The Kirkpatrick cooling schedule can more easily converge to local optima than the one proposed by Aars and Laarhoven (1985), especially when the constant used is small. However it allows for convergence in a reasonable number of iterations. In this work we will be using Kirkpatrick's approach:

$$
T_{S A_{i+1}}=\alpha T_{S A_{i}}
$$

where $T_{S A_{i 1}}$ and $T_{S A}$ are, respectively the new and the actual temperatures.

The suggested values for the constant, , are between 0.5 and 0.99. A global optimum can be achieved with high probabil- ity by considering constant values near 1 (Faber, Jockenhovel, \& Tsatsaronis, 2005). That corresponds to a slow cooling. Thus we only tested high values for , namely 0.95 and 0.9 . After performing some preliminary tests and looking to the solutions quality by comparison with the best solution found, 0.9 was chosen. The number of iterations per temperature was also tested; better results were obtained when using at least two times the number of optimization variables.

\subsection{Termination criteria}

Variouscriteria wereused tofinishiterations (Aars\& Laarhoven, 1985; Patel et al., 1991; Press et al., 1992).

We used simultaneously a fix number of iterations and of successes. The number of successes in a normal situation will not be reached. To establish the maximum number of iterations we carried out several optimizations varying that number and looking for the improvement in the objective function value of the solutions; we concluded that 26 cycles were sufficient. Considering a higher number of cycles did not improve the value of the objectivefunction for the solutions obtained.

\subsection{Tabu search}

TS is a stochastic optimization method based on memory (Glover, 1986). The search begins with a randomly obtained initial viable solution that is used to generate other solutions. The best solution from the set of generated solutions if better than the initial one, is chosen as a new solution, to start a new cycle. When the best solution is not better than the initial one (beginning of the cycle), it is sent to the tabu list that is systematically actualized by a procedure of the type first in-first out. This memory is used to avoid local optima. Intensification strategies will allow the thoroughexploration of promising areas whilediversificationstrategies are used to perform search in unknown areas. The aspiration criterion is used to override the tabu list when a better solution (than all the others) is found. In the simplest way the aspiration criteria can retrieve that better solution from the tabu list and use it as a new starting solution. Fig. 2 shows the flowchart for the tabu search programme; next we analyze some more important details.

In order to apply $\mathrm{TS}$ to a problem it is necessary to perform the following steps:

- define the objective function;

- define the strategy to determine the neighbourhood solutions;

- define tabu lists;

- define the aspiration criterion;

- define the diversification strategy;

- define the intensification strategy;

- define the termination criteria.

When applying TS to a given problem a set of preliminary optimizations should be done in order to establish the value of some of the algorithms parameters.

\subsubsection{Strategy to find neighbourhood solutions}

There are several strategies to find the neighbourhood solutions. Accordingly to Lin and Miller (2004) that can be done by adding to the vector initial solution a vector random increment, vc, determined by multiplying a vector of random numbers (between -1 and 1) and the vector magnitude of the variables vc search space; the random selection of a subset of possible changes was the criterion used by Cavin et al. (2005). The first method was selected because it is the most suitable for the problems studied (optimization that involves mostly continuous variables). 


\subsection{Tabu lists}

Specific solutions were saved in the tabu lists but the areas surrounding each of them are classified as tabu since in a continuous space the probability of visiting the same solution twice is very small. The tabu area is approximately $20 \%$ of the search space (centred in the solution) along each dimension (Lin \& Miller, 2004).

\subsection{Aspiration criterion}

The method suggested by Lin and Miller (2004) was chosen for aspiration criteria. It achieves a balance between intensification and diversification important in this case because the number of iterations will not be very high. It is based on a sigmoid function:

$$
s(k)=\frac{1}{1+\mathrm{e}^{-\sigma\left(k-k_{(\mathrm{cote})} \times M\right)}}
$$

where $k$ is the actual iteration number, $k_{\text {center }}$ determines when the sigmoid function is equal to $0.5, M$ is the total number of iterations ad is related to $M$. Its recommended range is between $5 / M$ and 10/M; in this workweused $5 / M$.

\subsection{Diversification strategy}

When in the tabu frequency list the frequency of a solution attains the maximum frequency (number of elements in the list) the search process is reinitialized by generating a new random solution (Lin and Miller, 2004).

\subsection{Intensification strategy}

The intensification strategy consists on choosing the best solution of the long memory list as a new starting solution after a certain number of iterations (Wang, Quan, \& Xu, 1999).

\subsection{Termination criterion}

The number of iterations as well as the number of solutions to be evaluated in each iteration was studied by performing some preliminary tests. From the results we could conclude that considering three solutions evaluation per iteration leads to good results.

Thus in each iteration we have three solutions, one of them coming from the previous iteration, except in the case when there is a random generation of the starting solution. For this reason a number of iterations equal to one-third of the number of iterations used inSA were used. Thiswasalsoconfirmed by doing runswith alarger number of iterations where no results improvement was observed.

\subsection{Objective functions}

Four objective functions were considered, later described in detail:

- Net Present Value (NPV1) - maximization;

- Net Present Value with inclusion of environmental impact costs (NPV2) - maximization;

- Global Potential Environmental Impact (GPEI) - minimization;

- Global Potential Environmental Impact according to EPS methodology (GPEI-EPS) - minimization.

\subsection{Economic type}

Net Present Value (NPV1) was used as economic objective function (Peters, Timmerhaus, \& West, 2003).

$$
\mathrm{NPV} 1=\sum_{l=1}^{n}\left[\left(V_{l}-C_{l}-d\right) \times\left(1-\frac{\Phi}{100}\right)+d\right] \times \frac{1}{\left(1+\frac{i}{\omega 0}\right)^{l}}-1 \text { (4) }
$$

where $V_{l}$ is the annual income in the year $l, C_{l}$ is the total annual cost, $d$ is the lineardepreciation, is theincometax, $i$ is theinterest (constant), $I$ is the total capital investment (entirely realized in year 0 ) and $n$ is the service life.

\subsection{Environmental type}

A suitable way to quantify environmental performance is through the consideration of the potential environmental impacts whose evaluation can be done by several methodologies. They imply the definition of the scope (space and time), the choice of environmental impacts category, their characterization, normalization and weighting to obtain an aggregated indicator. The environmental evaluation should be compatible with the available information so a gate to gate approach was taken, considering only the impacts originated by the process and by its energy requirements and a year of operation as the basis to do the calculations. Two methods were used: one based on the Guinée (2002) and on the IChemE (2007) methodologies and the other the Environmental Priority Strategies (EPS) methodology (Steen, 1999).

\subsubsection{Methodology based on Guinée E IChemE (GPEI)}

The chosen impact categories belong to the first set, namely the baseline impact categories and for which there is a characterization method available in the literature (Guinée, 2002) and are the following: depletion of abiotic resources, impact of land use, climate change, stratospheric ozone depletion, human toxicity, ecotoxicity, photo-oxidant formation, acidification and eutrophication. For the case of human toxicity there is no impact factor internationally accepted being more suitable to consider a characterization more linked to the process under study. For this reason we used the impact category from IChemE (2007), human health - carcinogenic, since in the process studied there are carcinogenic chemicals.

The normalization was done using a procedure similar to the WAR algorithm (Young \& Cabezas, 1999). The normalization within each category allows for a more independent analysis from spatial and temporal references which in the case of process optimization can have some advantages, since sometimes that information may not exist. The weight for each category was considered equal to 1 , since there is no location defined.

\subsubsection{EPS methodology (GPEI-EPS)}

The EPS system (Steen, 1999) was created to meet the requirements of product development and it takes into account the ISO14042 norm. There is an index for each element (e.g. $\mathrm{CO}_{2}$ expressed in environmental load unit (ELU)/ $\mathrm{kg}$ or transport expressed in ELU/(t.km)) which allows for the calculation of the total environmental load by summing all the environmental loads (element index times mass flow in the case of a chemical). This system is based on the willingness to pay concept, aiming at obtaining a figure easily understandable by the designers and by the society.

\subsection{Mixed type}

The mixed type objective function was constructed by considering a NPV that includes GPEI costs evaluated by the EPS methodology, since one ELU is approximately one euro (Tanzil et 


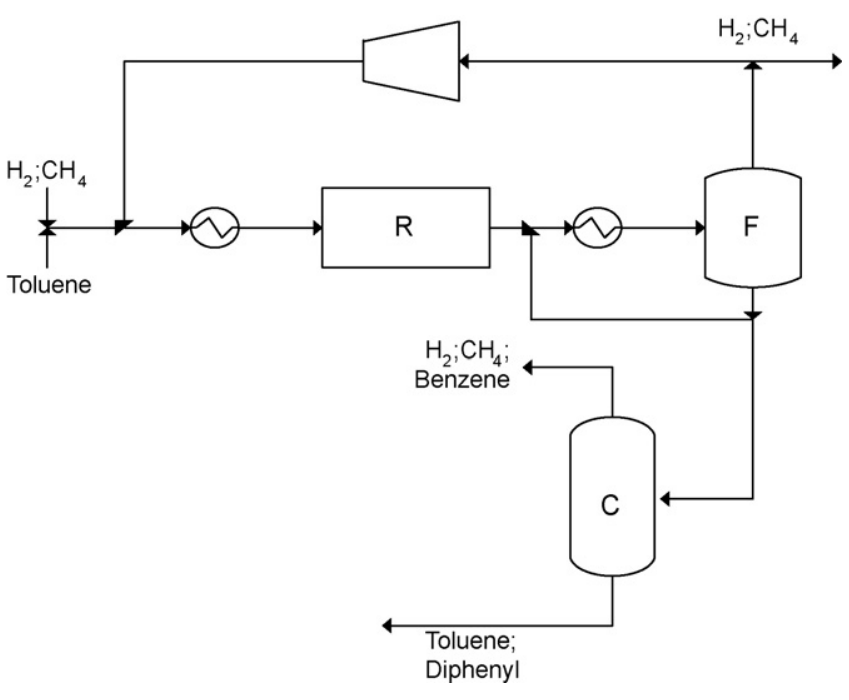

Fig. 3. Topology A.

al., 2002). The total annual cost is now calculated as the sum of $C_{l}$ plus $C_{A}$, where $C_{A}$ is the global potential environmental impact cost.

\section{Case study}

The non-catalytic hydrodealkylation of toluene to benzene (HDA) was selected as a case study mainly because it is quite well documented and several topologies with more or less complexity can be established. These different topologies are closely related to mass and heat conservation by reuse and recycling and thus with pollution prevention.

The reactions of interestare

$$
\begin{aligned}
& \mathrm{C}_{7} \mathrm{H}_{8}+\mathrm{H}_{2} \rightarrow \mathrm{C}_{6} \mathrm{H}_{6}+\mathrm{CH}_{4} \\
& 2 \mathrm{C}_{6} \mathrm{H}_{6} \Leftrightarrow \mathrm{C}_{12} \mathrm{H}_{10}+\mathrm{H}_{2}
\end{aligned}
$$

The raw-material streams are toluene and hydrogen with 3\% of methane at $3.964 \times 10^{6} \mathrm{~Pa}$ and $303.2 \mathrm{~K}$, normally used in industry and referred by Douglas (1988), Luyben, Tyréus, and Luyben (1999) and Qiu, Krishnaswamy, and Rangaiah (2000). The product (benzene) should be of high purity (higher than 99.0\%).

\subsection{Topologies}

The hierarchical heuristic procedures in conceptual design usually lead to a set of feasible flowsheets that should be further evaluated.Statistical design was introduced in those procedures to reduce the number of flowsheets to be studied. Two types of criteria were used: quality requirements and economic or environmental or mixed criterion. When the quality requirement was used only a small decrease of the number of topologies was observed (30-21). When economic, environmental or mixed criteria were used a large reduction: 21 topologies to five topologies (Martins \& Costa, 2008). These five topologies were considered for optimization and their schematic representations are presented in the following figures (Figs. 3-7) (Table1).

\subsection{Optimization variables}

The optimization variables were chosen mainly considering the characteristics of the process and of the topology and the variable input set determined by the simulator. Since we used a process simulator to model the process, degrees of freedom analysis was based on the available input set of variables established by the simulator.

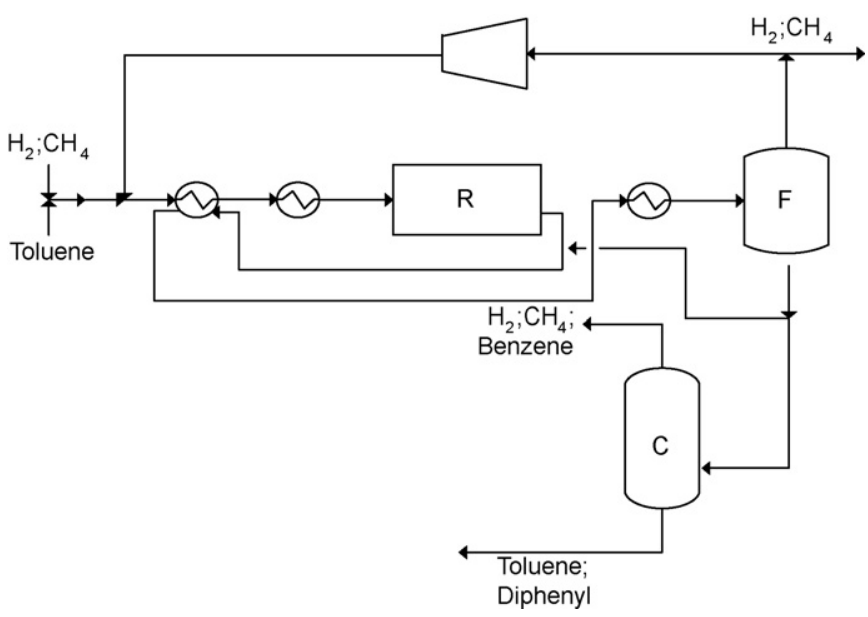

Fig. 4. Topology Ae.

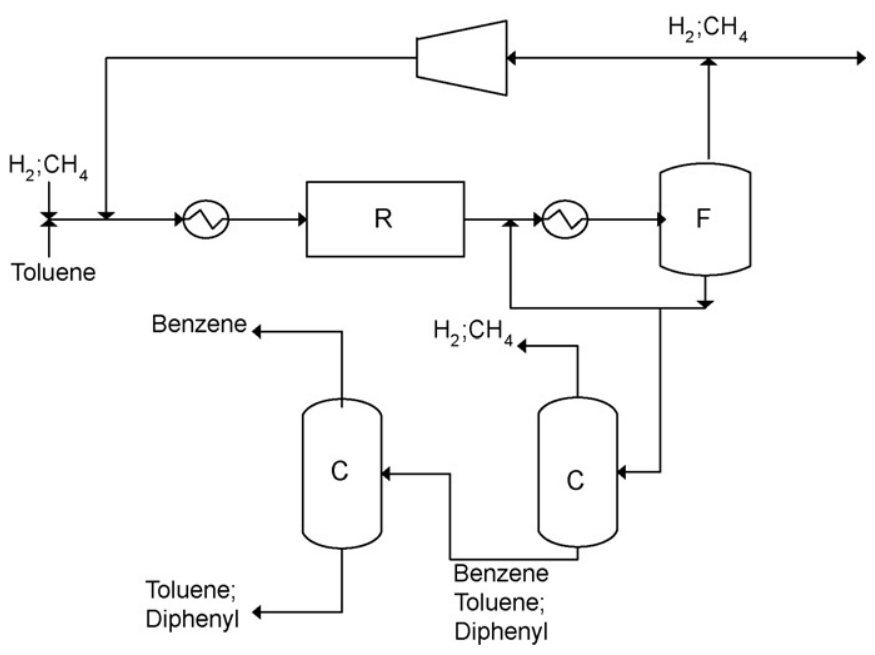

Fig. 5. Topology E.

For each piece of equipment considered, the simulator automatically carries a degrees of freedom analysis for that unit. For example when considering a column, the simulator will ask for the number of trays so it is possible to give different values to this integer variable and it can be considered an optimization variable.

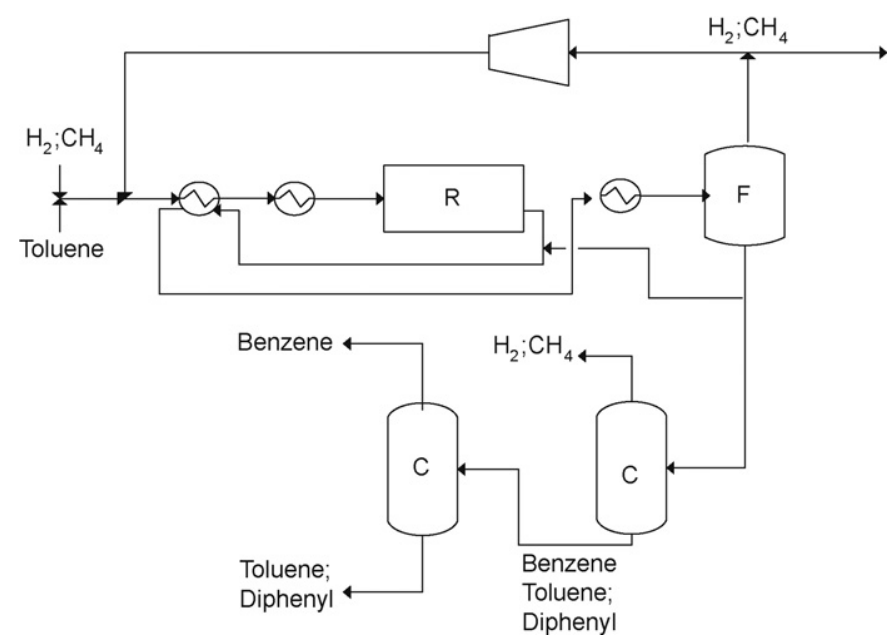

Fig. 6. Topology Ee. 

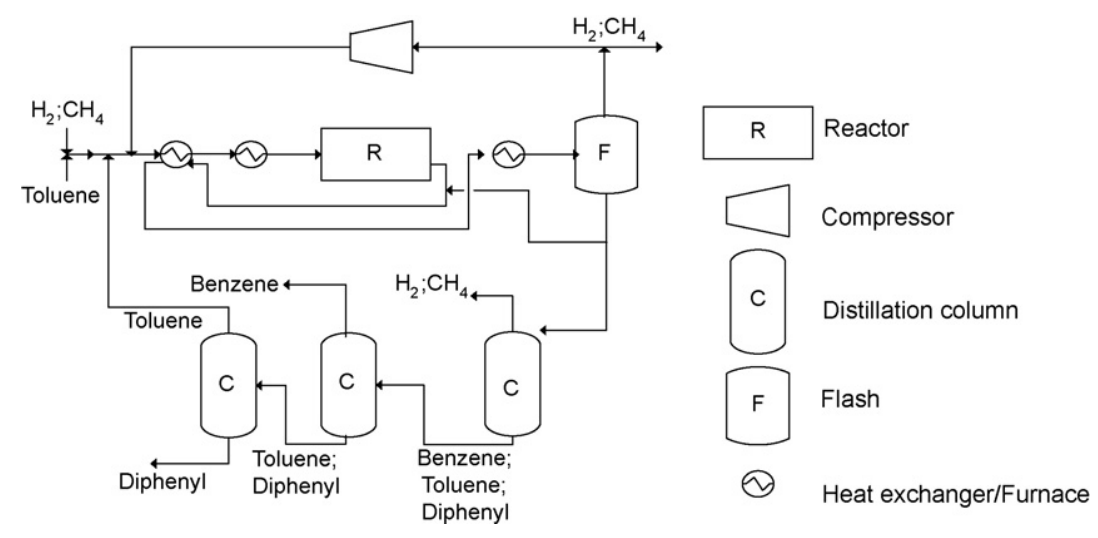

Fig. 7. Topology Ge.

Table 1

Equipment and characteristics of each topology.

\begin{tabular}{|c|c|c|c|c|c|c|c|c|}
\hline Topology & Adiabatic reactor & Flash & Compressor & Colunm $1^{\mathrm{a}}$ & Colunm $2^{\mathrm{b}}$ & Colunm $3^{c}$ & Toluene recirculation & Energy integration \\
\hline A & 1 & 1 & 1 & & 1 & & No & No \\
\hline $\mathrm{Ae}$ & 1 & 1 & 1 & & 1 & & No & Yes \\
\hline E & 1 & 1 & 1 & 1 & 1 & & No & No \\
\hline $\mathrm{Ee}$ & 1 & 1 & 1 & 1 & 1 & & No & Yes \\
\hline $\mathrm{Ge}$ & 1 & 1 & 1 & 1 & 1 & 1 & Yes & Yes \\
\hline
\end{tabular}

a Separation hydrogenmethane $\backslash$ benzenetoluenediphenyl.

b Separation hydrogenmethane \benzene \toluenediphenyl or hydrogenmethane benzene \toluenediphenyl or benzene $\backslash$ toluenediphenyl.

c Separation toluene $\backslash$ diphenyl.

In the simulation mode (the optimization process is continuous and for each iteration the simulator runs with the values of the optimization variables of that iteration) the simulator solves a problem where all the variables are specified, although some of them will be varied in each iteration. So degrees freedom analysis is imbedded in the simulator. Some of the available variables can be fixed (so this will decrease the number of degrees of freedom) using practical experience or process constrains. In the present case these were the following:

Table 2

Range for the optimization variables.

\begin{tabular}{|c|c|c|c|c|}
\hline Variables & Maximum & Minimum & References & Topologies \\
\hline Fresh reactant molar ratio & 2.50:1.00 & 1.00:1.00 & & $\mathrm{A}, \mathrm{Ae}, \mathrm{E}, \mathrm{Ee}, \mathrm{Ge}$ \\
\hline Flash pressure $(\mathrm{Pa})$ & $3.4 \times 10^{6}$ & $3.4 \times 10^{5}$ & $3.4 \times 10^{5} \mathrm{~Pa}$ (Douglas, 1988 ); $3.4 \times 10^{6}$ psia (Luyben et al., 1999) & $\mathrm{A}, \mathrm{Ae}, \mathrm{E}, \mathrm{Ee}, \mathrm{Ge}$ \\
\hline Benzene molar fraction in the purge & 0.01000 & 0.00500 & 0.0077 (Qiu et al., 2000) & $\mathrm{A}, \mathrm{Ae}, \mathrm{E}, \mathrm{Ee}, \mathrm{Ge}$ \\
\hline Purge flowrate (mol/s) & 72.5 & 12.6 & & $\mathrm{~A}, \mathrm{Ae}, \mathrm{E}, \mathrm{Ee}, \mathrm{Ge}$ \\
\hline Reactor volume $\left(\mathrm{m}^{3}\right)$ & 142 & 85 & $116 \mathrm{~m}^{3}$ (Douglas, 1988) & $\mathrm{A}, \mathrm{Ae}, \mathrm{E}, \mathrm{Ee}, \mathrm{Ge}$ \\
\hline Column 2 top stream toluene molar fraction & 0.0010 & 0.0003 & & $\mathrm{~A}, \mathrm{Ae}, \mathrm{E}, \mathrm{Ee}$ \\
\hline Column 2 top stream benzene recovery & 0.999 & 0.990 & & Ge \\
\hline Column 2 bottom stream benzene molar fraction & 0.0010 & 0.0003 & 0.0003 (Douglas, 1988; Luyben et al., 1999; Qiu et al., 2000) & $\mathrm{A}, \mathrm{Ae}, \mathrm{E}, \mathrm{Ee}$ \\
\hline Column 2 bottom stream toluene recovery & 0.999 & 0.990 & & $\mathrm{Ge}$ \\
\hline Column 2 operating pressure $(\mathrm{Pa})$ & $1.0 \times 10^{6}$ & $1.0 \times 10^{5}$ & & $\mathrm{~A}, \mathrm{Ae}, \mathrm{E}, \mathrm{Ee}, \mathrm{Ge}$ \\
\hline Column 1 top stream benzene molar fraction & 0.10 & 0.06 & & $\mathrm{E}, \mathrm{Ee}$ \\
\hline Column 1 top stream methane recovery & 0.999 & 0.990 & & Ge \\
\hline Column 1 reflux ratio & 5 & 0.25 & & $\mathrm{E}, \mathrm{Ee}, \mathrm{Ge}$ \\
\hline Column 1 operating pressure $(\mathrm{Pa})$ & $1.0 \times 10^{6}$ & $1.0 \times 10^{5}$ & (Douglas, 1988) & $\mathrm{E}, \mathrm{Ee}, \mathrm{Ge}$ \\
\hline Column 3 bottom stream diphenyl recovery & 0.999 & 0.990 & & $\mathrm{Ge}$ \\
\hline Column 3 operating pressure $(\mathrm{Pa})$ & $1.0 \times 10^{6}$ & $1.0 \times 10^{5}$ & & Ge \\
\hline Column 1 number of trays & 10 & 3 & 5 (Qiu et al., 2000) & $\mathrm{E}, \mathrm{Ee}, \mathrm{Ge}$ \\
\hline Column 2 number of trays & 42 & 23 & 27 (Qiu et al., 2000) & $\mathrm{A}, \mathrm{Ae}, \mathrm{E}, \mathrm{Ee}, \mathrm{Ge}$ \\
\hline Column 3 number of trays & 12 & 5 & 7 (Qiu et al., 2000) & $\mathrm{Ge}$ \\
\hline Column 3 top stream toluene recovery & 0.999 & 0.990 & & $\mathrm{Ge}$ \\
\hline
\end{tabular}

Table 3

Impact categories, models and indicators.

\begin{tabular}{|c|c|c|c|c|}
\hline Impact categories & Scale & Model & Reference chemicals & Necessary or emitted compounds \\
\hline Climate change & Global & Guinée & $\mathrm{CO}_{2}$ & $\begin{array}{l}\mathrm{CO}_{2} \\
\mathrm{CH}_{4}\end{array}$ \\
\hline Acidification & Regional/local & Guinée & $\mathrm{SO}_{2}$ & $\begin{array}{l}\mathrm{NO}_{x} \\
\mathrm{SO}_{2}\end{array}$ \\
\hline Eutrophication & Local & Guinée & $\mathrm{PO}_{4}^{3-}$ & $\mathrm{NO}_{x}$ \\
\hline Photo-oxidant formation & Local & Guinée & Ethylene & $\begin{array}{l}\mathrm{C}_{6} \mathrm{H}_{6} ; \mathrm{CH}_{4} \\
\mathrm{C}_{7} \mathrm{H}_{8} ; \mathrm{SO}_{2}\end{array}$ \\
\hline Human health-carcinogenic & Local & IChemE & Benzene & $\mathrm{C}_{6} \mathrm{H}_{6}$ \\
\hline Depletion of abiotic resources & Global/regional/local & Guinée & Antimony & Fuel \\
\hline
\end{tabular}


Table 4

SA and TS optimization performance.

\begin{tabular}{|c|c|c|c|c|c|c|c|c|}
\hline \multirow[t]{3}{*}{ Topology-objective function } & \multicolumn{3}{|c|}{ Average deviation (\%) } & \multicolumn{5}{|l|}{ Optimal solutions } \\
\hline & \multicolumn{2}{|l|}{ SA } & TS & \multicolumn{2}{|l|}{ SA } & \multirow[b]{2}{*}{ Purity } & \multicolumn{2}{|l|}{ TS } \\
\hline & Opt & Best & Best & Opt & Best & & Opt & Purity \\
\hline A-NPV1(MD) & 4.9 & 0.8 & 2.2 & -150 & & 0.941 & -148 & 0.941 \\
\hline A-NPV2 (MD) & 5.3 & 1.7 & 3.9 & -203 & & 0.939 & -200 & 0.951 \\
\hline A-GPEI & 26.8 & 4.9 & 10.4 & $2.21 \times 10^{5}$ & & 0.951 & $2.05 \times 10^{5}$ & 0.949 \\
\hline A-GPEI-EPS & 10.1 & 3.5 & 6.4 & $1.54 \times 10^{7}$ & & 0.963 & $1.47 \times 10^{7}$ & 0.959 \\
\hline Ae-NPV1(MD) & 2.7 & 1.3 & 2.6 & -144 & & 0.947 & -143 & 0.944 \\
\hline Ae-NPV2(MD) & 6.6 & 3.2 & 2.0 & & -190 & 0.937 & -194 & 0.941 \\
\hline Ae-GPEI & 10.1 & 4.1 & 2.6 & & $2.48 \times 10^{5}$ & 0.938 & $2.53 \times 10^{5}$ & 0.965 \\
\hline Ae-GPEI-EPS & 14.2 & 3.2 & 7.9 & $1.49 \times 10^{7}$ & & 0.957 & $1.46 \times 10^{7}$ & 0.956 \\
\hline E-NPV1(MD ) & 13.1 & 1.0 & 2.1 & & -157 & 0.999 & -156 & 1.000 \\
\hline E-NPV2(MD) & 7.0 & 4.9 & 2.9 & -209 & & 1.000 & -206 & 1.000 \\
\hline E-GPEI & 28.0 & 15.3 & 4.4 & $1.89 \times 10^{5}$ & & 1.000 & $1.98 \times 10^{5}$ & 0.999 \\
\hline E-GPEI-EPS & 19.9 & 6.9 & 5.5 & & $1.53 \times 10^{7}$ & 0.999 & $1.53 \times 10^{7}$ & 1.000 \\
\hline Ee-NPV1(MD ) & 148.2 & 137.7 & 6.7 & -178 & & 0.999 & -175 & 0.999 \\
\hline Ee-NPV2(MD) & 151.5 & 151.5 & 144.8 & -225 & & 0.999 & -214 & 0.999 \\
\hline Ee-GPEI & 244.1 & 244.1 & 141.0 & $3.51 \times 10^{5}$ & & 0.999 & $3.19 \times 10^{5}$ & 1.000 \\
\hline Ee-GPEI-EPS & 31.7 & 29.2 & 98.0 & $3.60 \times 10^{7}$ & & 0.999 & $1.69 \times 10^{7}$ & 1.000 \\
\hline Ge-NPV1(MD) & 5.0 & 2.2 & 8.4 & & -154 & 0.997 & -148 & 0.998 \\
\hline Ge-NPV2(MD) & 15.9 & 8.9 & 10.2 & & -198 & 0.999 & -198 & 0.991 \\
\hline Ge-GPEI & 31.7 & 14.6 & 5.9 & & $2.50 \times 10^{5}$ & 0.998 & $2.59 \times 10^{5}$ & 1.000 \\
\hline Ge-GPEI-EPS & 21.0 & 17.7 & 16.4 & & $1.48 \times 10^{7}$ & 0.997 & $1.44 \times 10^{7}$ & 0.996 \\
\hline
\end{tabular}

Opt: optimum value of the objective function; Best: best value of the objective function; Purity: benzene molar fraction in the product.

- Reactor feed temperature $=894 \mathrm{~K}$ (below this temperature the reaction is too slow and above $978 \mathrm{~K}$ undesired reactions occur (Douglas, 1988)).

- Cooling water temperature $=293 \mathrm{~K}$ (inlet); $313 \mathrm{~K}$ (outlet).

- Reactor pressure $=3.4 \times 10^{6} \mathrm{~Pa}$ (Douglas, 1988).

- Stream temperature after cooling in the heat exchanger placed after the reactor $=311 \mathrm{~K}$ (Douglas, 1988).

- Coolant flowrate for rapid cooling of the outlet stream of the reactor to avoid coke formation $=19.6 \mathrm{~mol} / \mathrm{s}$ (Luyben et al., 1999).

- Minimum temperature difference in the heat exchangers $=5 \mathrm{~K}$

(Biegler, Grossmann, \& Westerberg, 1997).

- Compressor outlet pressure $=3.8 \times 10^{6} \mathrm{~Pa}$ (Douglas, 1988).

In the simulation of the distillation columns we considered partial condensers and kettle reboilers.

Table 2 indicates the optimization variables for each topology. For Ge topology component recoveries (= component molar flowrate in the selected stream/component molar flowrate in the column feed stream) were used instead of molar fractions since the simulator performed better this way.

The range for the optimization variables was established using data available in the literature (Douglas, 1988; Luyben et al., 1999; Qiu et al., 2000) and by doing some preliminary calculations.

Table 2 presents the boundaries for each optimization variable.

\subsection{Impact categories}

The impact related to land use was not considered since at this stage neither the area needed for the plant nor its location is known. Also there are no compounds for the impact category stratospheric ozone depletion. The impact category human toxicity from the Guinée model (Guinée, 2002) was replaced by the impact category human health - carcinogenic from IChemE (IChemE, Janeiro/2007). The impact category ecotoxicity was not considered since the factors for the chemicals present in this process are very low and for that reason their impact would not be meaningful.

Table 3 resumes impact categories, models and indicators that wereused.

\section{Results}

SA and tabu searchoptimizationmethodologiesusually find different optima for each run although the values of the objective function are not too different. So there is a certain dispersion of results, meaning that smaller dispersion increases the chance of obtaining a result closest to the best (global optimum) when performing only one run. Thus a way to assess performance can be based on observed dispersion measured in the present case by an average deviation. For SA two types of deviation where calculated: the average deviation relatively to the optimumsolution found in all SA runs for the same topology and objective function and the average deviation relatively to the best solution found. For tabu search only the first average deviation was evaluated. For each topology, algorithm and objective function five runs were performed. The SA algorithm always stores the best solution ever found during the optimization and not only thelast solution. Table 4 shows the results.

Retaining the best solution ever found in SA is a good strategy since the average deviation is normally smaller than when the optimum solution is considered.

We consider that an algorithm has good performance (bold in Table 4) when the average deviation is equal or smaller than $5 \%$ and reasonable performance (italic in Table 4) for average deviations between 5 and $10 \%$.

SA presented good performance for topologies A and Ae whichever the objective function, for topology E with NPV type objectivefunctions and for topology Gefor theNPV1 objectivefunction. Reasonable performancewas observed for topologies EandGe with GPEI-EPS and NPV2 objective functions, respectively. In the remaining cases the performance is poor, especially for topology Ee.

TS presented good performance for topologies $\mathrm{A}, \mathrm{Ae}$ and $\mathrm{E}$ when the objective functions are of the NPV type and also for topologies Ae and E with GPEI objective function. Reasonable performance was observed for topology A with GPEI and GPEI-EPS objective functions, for topologies Ae and E with the GPEI-EPS objective function, for topology Ee with NPV1 objective function and for topology Ge 


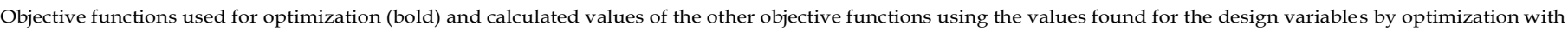
the chosen objective functions.

\begin{tabular}{|c|c|c|c|c|c|c|c|c|c|c|}
\hline \multirow[t]{2}{*}{ Objective function } & \multicolumn{2}{|l|}{ A } & \multicolumn{2}{|l|}{ Ae } & \multicolumn{2}{|l|}{$\mathrm{E}$} & \multicolumn{2}{|l|}{$\mathrm{Ee}$} & \multicolumn{2}{|l|}{$\mathrm{Ge}$} \\
\hline & SA & TS & SA & TS & SA & TS & SA & TS & SA & TS \\
\hline NPV1 $\times 10^{-8} \mathrm{D}$ & -1.50 & -1.48 & -1.44 & -1.43 & -1.57 & -1.56 & -1.78 & -1.75 & -1.54 & -1.48 \\
\hline $\mathrm{NPV} 2 \times 10^{-8} \mathrm{D}$ & -2.04 & -2.01 & -1.97 & -1.98 & -2.12 & -2.09 & -2.34 & -2.40 & -2.04 & -1.97 \\
\hline GPEI $\times 10^{-5}$ & 2.15 & 2.08 & 2.62 & 2.83 & 2.49 & 2.35 & 3.31 & 3.60 & 4.70 & 3.40 \\
\hline GPEI-EPS $\times 10^{-7}$ & 1.64 & 1.58 & 1.57 & 1.65 & 1.65 & 1.59 & 1.70 & 1.95 & 1.51 & 1.46 \\
\hline $\mathrm{NPV} 1 \times 10^{-8} \mathrm{D}$ & -1.51 & -1.51 & -1.40 & -1.44 & -1.56 & -1.55 & -1.70 & -1.63 & -1.48 & -1.55 \\
\hline $\mathbf{N P V} 2 \times 10^{-8} \mathrm{D}$ & -2.03 & -2.00 & -1.90 & -1.94 & -2.09 & -2.06 & -2.25 & -2.14 & -1.98 & -1.98 \\
\hline $\mathrm{GPEI} \times 10^{-5}$ & 2.23 & 2.05 & 2.50 & 2.51 & 2.46 & 2.22 & 3.23 & 2.97 & 3.45 & 6.09 \\
\hline GPEI-EPS $\times 10^{-7}$ & 1.54 & 1.46 & 1.51 & 1.49 & 1.59 & 1.55 & 1.66 & 1.54 & 1.51 & 1.30 \\
\hline $\mathrm{NPV} 1 \times 10^{-8} \mathrm{D}$ & -1.48 & -1.49 & -1.43 & -1.52 & -1.71 & -1.73 & -1.84 & -1.90 & -1.89 & -1.81 \\
\hline $\mathrm{NPV} 2 \times 10^{-8} \mathrm{D}$ & -1.98 & -1.99 & -1.93 & -2.04 & -2.57 & -2.54 & -2.47 & -2.47 & -3.03 & -2.80 \\
\hline GPEI $\times 10^{-5}$ & 2.21 & 2.05 & 2.48 & 2.53 & 1.89 & 1.98 & 3.51 & 3.19 & 2.50 & 2.59 \\
\hline GPEI-EPS $\times 10^{-7}$ & 1.52 & 1.51 & 1.51 & 1.58 & 2.59 & 2.44 & 1.91 & 1.72 & 3.47 & 3.01 \\
\hline $\mathrm{NPV} 1 \times 10^{-8} \mathrm{D}$ & -1.58 & -1.57 & -1.48 & -1.46 & -1.53 & -1.61 & -2.56 & -1.70 & -1.68 & -1.50 \\
\hline $\mathrm{NPV} 2 \times 10^{-8} \mathrm{D}$ & -2.09 & -2.06 & -1.97 & -1.94 & -2.04 & -2.12 & -3.75 & -2.26 & -2.17 & -1.98 \\
\hline $\mathrm{GPEI} \times 10^{-5}$ & 2.44 & 2.23 & 2.56 & 2.53 & 2.28 & 2.45 & 6.68 & 3.30 & 5.64 & 4.67 \\
\hline GPEI-EPS $\times 10^{-7}$ & 1.54 & 1.47 & 1.49 & 1.46 & 1.53 & 1.53 & 3.60 & 1.69 & 1.48 & 1.44 \\
\hline
\end{tabular}

with NPV1, NPV2 and GPEI objective functions. Resuming we can say that the algorithms are not efficient for more complex topologies (e.g. Ee and Ge) particularly with environmental impact type objective functions and that in average and from this point of view SA performs better than TS.

The process we are using as case study aims at producing high purity benzene (purity above 0.990 ). From Table 4 we can see that only topologies E, Ee and Ge are able to reach that stage, due to the addition of a second distillation column. During optimizations with topologies $\mathrm{A}$ and Ae we found a few solutions leading to purities higher than 0.990 . These were not retained because conducted to objective functions values worse than those observed for topologies E and Ge. The reason for this relies in increased capital costs and energy consumption due to higher streams flowrates (e.g. gas recirculation system). Usually the use of global impact type objective functions leads to better purities. From this point a view both algorithms show almost similar performance, with a marginal advantage for TS.

We take now the perspective of choosing a topology based on the value of an objective function. For NPV1 and NPV2 the best topology is Ae for both SA and TS, although high purity cannot be reached for the final solutions. This is possibly due to notconsidering a penalty for benzene price when purity is lower than 0.990 . If this was the case Ge topology would possibly had been elected. In the case of GPEI and of GPEI-EPS both algorithms conducted to the same topologies, respectively E and Ge. Topology Ee got the worse values for all objective functions. This is essentially linked to high flowrates that are not economically neither environmentally favourable.

Comparing now the values of the objective functions obtained by SA and TS for each topology we can conclude that from this point of view TS performed quite better than SA, although those values were not too different.

If we choose an objective function and optimization method to screen among various topologies, how invariant is this choice to using other objective functions. Table 5 shows the value of other objective functions calculated with the optimum values for the design variables obtained by optimization with the chosen objective function(bold).

When using SA with NPV1 as objective function the best topology is Ae. Calculated NPV2 points to the same choice but GPEI gives topology E and GPEI-EPS, topology Ge. TS gives similar results. If we look to other objective functions we get results in the same line as described. So we can conclude that NPV1 and NPV2 give very

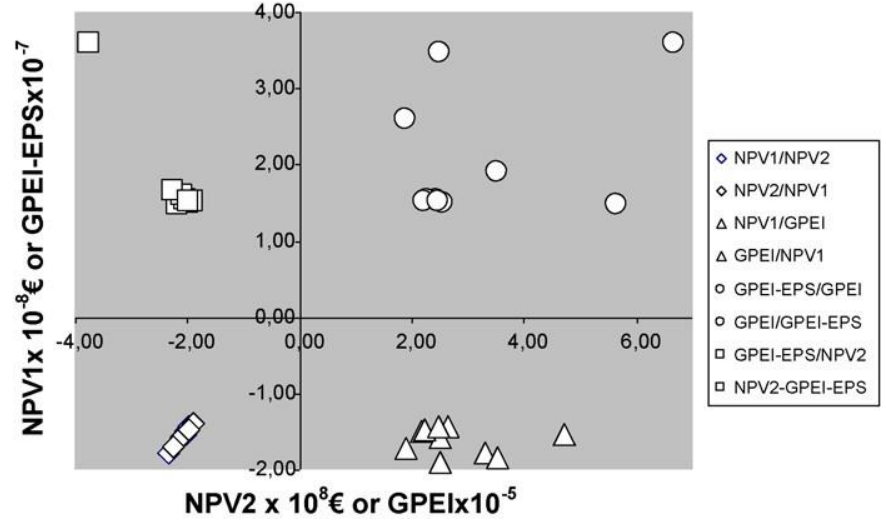

Fig. 8. Comparison among various objective functions when using SA.

close results in terms of selecting a topology. Taking now the environmental potential impact type objective functions we can easily conclude that topology selection is not invariant with the choice of this kind of objective function.

Another issue is the possibility of the existence of trade-offs when using different objective functions. Figs. 8 and 9 compare several objective functions: NPV1 with GPEI (2nd quadrant) and NPV2 (3rd quadrant), GPEI with GPEI-EPS (1st quadrant) and NPV2 with GPEI-EPS (4th quadrant), respectively for optimizations using SA and TS.

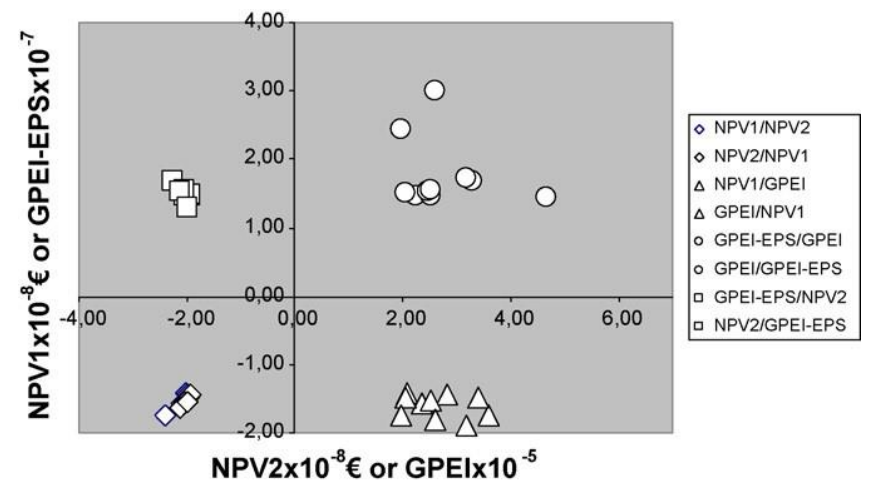

Fig. 9. Comparison among various objective functions when using TS. 
Table 6

Some optimum values for the design variables.

\begin{tabular}{|c|c|c|c|c|c|c|c|c|}
\hline \multirow[t]{2}{*}{ Design variables } & \multirow{2}{*}{$\frac{\mathrm{NPV1}(\mathrm{Ae})}{\mathrm{SA}}$} & \multirow[b]{2}{*}{ TS } & \multirow{2}{*}{$\begin{array}{l}\text { NPV2 (Ae) } \\
\text { SA }\end{array}$} & \multirow[b]{2}{*}{ TS } & \multirow{2}{*}{$\begin{array}{l}\text { GPEI(E) } \\
\text { SA }\end{array}$} & \multirow[b]{2}{*}{ TS } & \multirow{2}{*}{$\begin{array}{l}\text { GPEI-EPS (Ge) } \\
\text { SA }\end{array}$} & \multirow[b]{2}{*}{ TS } \\
\hline & & & & & & & & \\
\hline $\mathrm{vc}(0)$ & 1.601 & 1.751 & 1.675 & 1.626 & 1.331 & 1.101 & 1.467 & 1.126 \\
\hline $\mathrm{vc}(3)$ & $2.63 \times 10^{6}$ & $2.95 \times 10^{6}$ & $3.35 \times 10^{6}$ & $3.05 \times 10^{6}$ & $3.11 \times 10^{6}$ & $2.45 \times 10^{6}$ & $7.05 \times 10^{5}$ & $3.05 \times 10^{6}$ \\
\hline $\mathrm{vc}(4)$ & 0.0076 & 0.0055 & 0.0073 & 0.0087 & 0.0071 & 0.0054 & 0.0052 & 0.0060 \\
\hline $\mathrm{vc}(5)$ & 59.9 & 65.2 & 62.3 & 59.4 & 58.1 & 48.5 & 52.1 & 39.1 \\
\hline $\operatorname{vc}(6)$ & 92.9 & 87.8 & 94.5 & 124.6 & 108.7 & 138.8 & 102.0 & 107.6 \\
\hline $\operatorname{vc}(7)$ & 0.00057 & 0.00030 & 0.00071 & 0.00095 & 0.00043 & 0.00099 & 0.99252 & 0.99499 \\
\hline $\mathrm{vc}(8)$ & 0.00070 & 0.00037 & 0.00039 & 0.00042 & 0.00050 & 0.00045 & 0.99459 & 0.99200 \\
\hline $\operatorname{vc}(9)$ & $3.27 \times 10^{5}$ & $9.00 \times 10^{5}$ & $2.34 \times 10^{5}$ & $1.76 \times 10^{5}$ & $5.32 \times 10^{5}$ & $5.79 \times 10^{5}$ & $5.97 \times 10^{5}$ & $4.03 \times 10^{5}$ \\
\hline $\mathrm{vc}(10)$ & & & & & 0.089 & 0.089 & 0.992 & 0.994 \\
\hline $\mathrm{vc}(11)$ & & & & & 2.62 & 1.80 & 0.63 & 1.15 \\
\hline $\mathrm{vc}(12)$ & & & & & $8.58 \times 10^{5}$ & $5.90 \times 10^{5}$ & $5.03 \times 10^{5}$ & $3.21 \times 10^{5}$ \\
\hline $\mathrm{vc}(13)$ & & & & & & & 0.99423 & 0.99202 \\
\hline $\operatorname{vc}(14)$ & & & & & & & $3.18 \times 10^{5}$ & $1.02 \times 10^{6}$ \\
\hline $\mathrm{vc}(15)$ & & & & & 9 & 9 & 4 & 3 \\
\hline $\mathrm{vc}(16)$ & 38 & 39 & 32 & 35 & 29 & 40 & 34 & 29 \\
\hline $\mathrm{vc}(18)$ & & & & & & & 7 & 8 \\
\hline $\mathrm{vc}(19)$ & & & & & & & 0.99792 & 0.99300 \\
\hline
\end{tabular}

The observation of these figures shows that there is no evidence of trade-offs in the sense of having one objective function increasing while the other decreases. We also can conclude that NPV1 is quite independent of GPEI (2nd quadrant), NPV2 and GPEI-EPS (4th quadrant) almost do not vary, NPV1 and NPV2 (3rd quadrant) display some proportionality and GPEI and GPEI-EPS (1st quadrant) also demonstrate some proportionality although the data is quite scattered.

Since no trade-offs were observed it is also not possible to see if the combination of economic and environmental concerns in NPV2 could solve them.

Table 6 shows the optimum values of the design variables for each objective function best topology and algorithm. These values vary with the objective function and algorithm even in the cases where the objective functions have values quite close. Reactor volume varies between 87.8 and $138.8 \mathrm{~m}^{3}$ and columns 1,2 and 3 number of stages varies, respectively between 29 and 40, 3 and 9 and 7 and 8 . Molar fractions, recoveries, reflux ratios and streams flowrates also vary.

\section{Conclusions}

This work analyzed the behaviour of two optimization algorithms, simulated annealing and tabu search, combined with four objective functions: two of the net present value type, one of them including environmental costs and two of the global potential impact type. The hydrodealkylation of toluene to produce benzene was used as case study, considering five topologies with different complexities mainly obtained by including or not liquid recycling and heat integration.

The performance of the algorithms together with the objective functions was observed from various angles:

(a) both optimization methodologies usually show a certain dispersion of results that can be assessed by an average deviation, smaller dispersion meaning better performance.

We concluded that both algorithms performance was poor for more complex topologies, particularly with environmental impact type objective functions and that in average and from this point of view SA (with memory) performs better than TS.

(b) For this case study we aim at producing high purity benzene. This can only be reached with reasonable economic and environmental impacts (costs) when using topologies with at least two distillation columns.
Generally we can say that impact type objective functions lead to higher purities and that, from this point of view, the algorithms have essentially the same performance.

(c) When using hierarchical heuristic procedures in process conceptual design it is common to reach a point where a small number of topologies need to be screened and one of them chosen, for example based on the value of an objective function.

NPV type objective functions conducted to the choice of similar topologies and the same was observed for GPEI type objective functions, although with the choice of other topologies. The values of the objective functions obtained by SA and TS were not too different, although TS performed quite better thanSA;

(d) We have to take one objective function to perform this screening process it would be interesting to know how dependent on that choice are the results.

In present case NPV type objective functions give very close results in terms of selecting a topology. This was not observed in the case of using environmental potential impact type objective functions that lead to results not only different from those obtained with NPV type objective functions but also different from one another.

(e) Since we are dealing with economic and environmental objective functions it is interesting to look for possible trade-offs, in the sense of having one objective function increasing while the other decreases.

We observed no evidence of this kind of trade-offs, despite the detection of some proportionality between NPV and between GPEI type objective functions.

(f) For best topologies the optimum values found for the design variables vary with algorithm and objective function, even in the cases where the objective functions have quite close values.

As main conclusions we can say that these algorithms should be carefully used with complex topologies since they shown difficulties in these cases, although SA performed better than TS for this aspect. For NPV and GPEI type objective functions both methods point to the same topologies although with the choice of different topologies. SA and TS obtained similar values for the objective functions, although TS performed quite better than SA. In the cases where the objective functions have quite close values the values found for the design variables vary. 


\section{References}

Aars, E. L. H., \& Laarhoven, P. J. M. (1985). Statistical cooling: A general approach to combinatorial optimization problems. Philippines Journal of Research, 40, 193-226.

Biegler, L. T., Grossmann, I. E., \& Westerberg, A. W. (1997). Systematic methods of chemical process design. New Jersey: Prentice-Hall, Inc.

Brennecke, J. F., \& Stadtherr, M. A. (2002). A course in environmentally conscious chemical process engineering. Computers $\mathcal{E}$ Chemical Engineering, 26, 307-318.

Cano-Ruiz, J. A., \& McRae, G. J. (1998). Environmentally conscious chemical process design. Annual Review of Energy and the Environment, 23, 499-523.

Cardoso, M. F., Salcedo, R. L., \& Azevedo, S. F. D. (1994). Nonequilibrium simulated annealing: A faster approach to combinatorial minimization. Industrial $\mathcal{E}$ Engineering Chemistry Research, 33, 1908-1918.

Cardoso, M. F., Salcedo, R. L., \& Azevedo, S. F. D. (1996). The simplex-simulated annealing approach to continuous non-linear optimization. Computers $\mathcal{E}$ Chemical Engineering, 20, 1065-1080.

Cardoso, M. F., Salcedo, R. L., Azevedo, S. F. D., \& Barbosa, D. (1997). A simulated annealing approach to the solution of minlp problems. Computers $\mathcal{E}$ Chemical Engineering, 21, 1349-1364.

Cavin, L., Fischer, U., Mosat, A., \&Hungerbuhler, K. (2005). Batch processoptimization in a multipurpose plant using Tabu search with a design-space diversification. Computers \& Chemical Engineering, 29, 1770-1786.

Das, H., Cummings, P. T., \& LeVan, M. D. (1990). Scheduling of serial multiproduct batch processes via simulated annealing. Computers $\mathcal{E}$ Chemical Engineering, 14, 1351-1362.

Dietz, A., Azzaro-Pantel, C., Pibouleau, L., \& e Domenech, S. (2006). Multiobjective optimization for multiproduct batch plant design under economic and environmental considerations. Computers \& Chemical Engineering, 30, 599-613.

Dolan, W. B., Cummings, P. T., \& Van, M. D. L. (1990). Algorithmic efficiency of simulated annealing for heat exchanger network design. Computers $\mathcal{E}$ Chemical Engineering, 14, 1039-1050.

Douglas, J. M. (1988). Conceptual design of chemical processes. Singapore: McGrawHill Chemical Engineering Series.

El-Halwagi, M. M., \& Manousiouthakis, V. (1990). Automatic Synthesis of MassExchange Networks with Single-Component Targets. Chem. Eng. Sci., 45(9) 2813-2831.

Faber, R., Jockenhovel, T., \& Tsatsaronis, G. (2005). Dynamic optimization with simulated annealing. Computers \& Chemical Engineering, 29, 273-290.

Glover, F. (1986). Future paths for integer programming and links to artificial intelligence. Computers and Operations Research, 13, 533-549.

Guinée, J. B. (2002). Handbook on life cycle assessment. Operational guide to the ISO standards. Dordrecht: Kluwer Academic Publishers.

Hanke, M., \& Li, P. (2000). Simulated annealing for the optimization of batch distillation processes. Computers \& Chemical Engineering, 24, 1-8.
Hertz, A., \& Widmer, M. (2003). Guidelines for the use of meta-heuristics in combinatorial optimization. European Journal of Operational Research, 151, 247-252.

IChemE (January, 2007). The sustainability metrics. www.icheme.org.

Kirkpatrick, S. C. D., Gelatt, J., \& Vecchi, M. P. (1983). Optimization by simulated annealing. Science, 220, 671-680.

Ku, H., \& Karimi, I. (1991). An evaluation of simulated annealing for batch process scheduling. Industrial \& Engineering Chemistry Research, 30, 163-169.

Lin, B., \& Miller, D. C. (2004). Tabu search algorithm for chemical process optimization. Computers \& Chemical Engineering, 28, 2287-2306.

Luyben, W. L., Tyréus, B. D., \& Luyben, M. L. (1999). Plantwide process control. New York: McGraw-Hill.

Martins, F., \& Costa, C. A. V. (2008). Conceptual design of sustainable processes. Using heuristics with stastistical design. In Proceedings of the 4th IASMENWSEAS international conference on ENERGY, ENVIRONMENT, ECOSYSTEMS and SUSTAINABLE DEVELOPMENT (EEESD'08) (ISBN: 978-960-6766-71-8) , pp. 484-494

Patel, A. N., Mah, R. S. H., \& Karimi, I. A. (1991). Preliminary design of multiproduc noncontinuous plants using simulated annealing. Computers \& Chemical Engineering, 15, 451-469.

Peters, M. S., Timmerhaus, K. D., \& West, R. E. (2003). Plant design and economics for chemical engineers. Boston: McGraw-Hill.

Pibouleau, L., Domenech,S., Davin, A., \&e Azzaro-Pantel,C. (2005). Expérimentations numériques sur les variantes et paramètres de la méthode du recuit simulé. Chemical Engineering Journal, 105, 117-130.

Press, W. H, \& Teukolsky, S. A. (1991). Simulated annealing optimization over continuous spaces. Computers in Physics, 426-429.

Press, W. H., Teukolsky, S. A., Vetterling, W. T., \& Flannery, B. P. (1992). Numerical recipes in C. New York: Cambridge University Press.

Qiu, Q. F., Krishnaswamy, P. R., \& Rangaiah, G.P. (2000). Modelling and simulation of HDA process for plant-wide design and control studies. In Chemical and Process Engineering Conference (CPEC) Singapore.

Simulation Sciences Inc. (1994-2005). ProII 71.

Smith, R. (1995). Chemical process design. Singapore: McGraw-Hill, Inc.

Steen, B. (1999). A systematic approach to environmental priority strategies in product development (EPS). Version 2000 - Models and data of the default method. Centre for the Environmental Assessment of Products and Material Systems.

Tanzil, D., Murphy, K., Schwarz, J., Restoske, M., Beloff, B., and Beaver, E. (2002). Incorporating total cost assessment methodology to enhance chemical complex optimization. Final Report. Gulf Coast Hazardous Substance Research Center (GCHSRC). BRIDGES to Sustainability. Houston, TX.

Wang, C., Quan, H., \& e Xu, X. (1999). Optimal design of multiproduct batch chemical process using Tabu search. Computers \& Chemical Engineering, 23, 427-437.

Young, D. M, \& Cabezas, H. (1999). Designing sustainable processes with simulation: The waste reduction (WAR) algorithm. Computers \& Chemical Engineering, 23, 1477-1491 